\title{
Ultraviolet inspection for diagnostics of coronary discharge on suspension insulators and lines of $35-110 \mathrm{kV}$
}

\author{
Phuong Nguyen ${ }^{1}$, , Sergey Dudkin ${ }^{1}$, Tamara Salova ${ }^{1}$, Karl Mertens ${ }^{1}$, Gennadiy \\ Cherkesov $^{1}$, Evgenii Yurevich $^{1}$, Rostislav Ivanovski ${ }^{1}$, Georgy Fokin ${ }^{1}$, and Sergey \\ Vokhmyanin $^{1}$ \\ ${ }^{1}$ Peter the Great St.Petersburg Polytechnic University, Polytechnicheskaya, 29, St. Petersburg, \\ 195251, Russia
}

\begin{abstract}
In this paper, the question of the correctness and sufficiency of using a UV camera for the diagnosis of insulators and $35-110 \mathrm{kV}$ power lines is considered. As a result of the literary analysis, controversial moments and minuses are identified in the existing method. In addition, for its refinement and correction, an experiment was conducted at the laboratory of the TVN $\mathrm{SPbPU}$. The analysis of the experiment showed features and nuances in the work of the camera, which must be taken into account in the UV survey.
\end{abstract}

\section{Introduction}

Evaluation of the technical condition, reliability of the insulation of electrical equipment is an actual problem. It is confirmed by experience and statistics of operation at power plants and railway facilities.

Monitoring the state of external insulation of electrical equipment is one of the main types of diagnostics in the operation of electrical networks and equipment, since insulation defects lead to the ionization of air voids and the generation of partial discharges or corona. These discharge processes gradually destroy insulation, cause energy losses and radio interference, etc [1]. In the practice of operation, the normal state of insulation is the absence of partial discharges and corona, and their presence indicates possible defects or contamination of insulation $[2,3]$. The need to determine the resistance to surface partial discharges (PCR) increases in the case of using new polymeric materials due to their properties $[4,5,6]$.

At the moment, contact (insulation resistance measurement, ultrasonic method, voltage measurement on insulators using a measuring rod) and non-contact (acoustic, thermal imaging, ultraviolet) methods are used for technical diagnostics of insulation $[7,8]$.

Non-contact diagnostic methods have an undoubted advantage over a contact one in the absence of necessity to disconnect a measurement object. In addition, UV and IR methods and can at all point to a probable defect, visualizing its place on the diagnosed object $[9,10]$.

According to Russian regulatory documents, at control of electrical equipment, there should be no visible corona discharges at the highest operating voltage. At their emergence

\footnotetext{
*Corresponding author: nguyenphuong95@mail.ru
} 
owing to damages to insulation of electric equipment UV-control allows to find them in due time.

Considering of the main methods of UV inspection [11, 12]:

1. Location of the centers of the corona category on power line wires [13], etc. EO.

2. Monitoring the state of suspension and supporting porcelain, glass and polymer insulation.

3. Assessment of the degree of contamination of insulation.

All methods are based on an estimate of the intensity of the discharge: the number of flashes, the size of the UV spot. Depending on the intensity of the outbreaks observed, the external insulation status of electrical equipment is assessed according to the 3 categories of defect development: developing, pre-emergency and emergency [14].

\section{Equipment}

For carrying out of UV-inspection the special chamber, capable to find out the centers of UVradiation is used. Consider a simplified scheme for the operation of the ultraviolet camera. A beam of light hits the prism: the part is refracted and goes to the video lens, and then to the first matrix; the other part, reflected twice, passes through the ultraviolet filter (the most important and expensive element of the camera) and is directed also to the lens and the matrix. The information about the light flux is then fed to the central processing unit of signals, where also two video signals are combined [9]. The output is a video signal with conditional flashes indicating the presence of bit activity.

UV-cameras from CoroCam and Ofil are widely used. These brands produce cameras that can be used in the daytime, when solar activity is high. In the PJSC "Rosseti", the cameras of both companies are very demanded. The experiments, described in this article were conducted using the Ofil DayCor Superb camera.

\section{Conditions influencing the indications of the UV camera}

The sensitivity of the camera detector is the most important characteristic determining the reliability of ultraviolet diagnostics. The UV range of the electromagnetic spectrum lies to the left of the visible range and is approximately 200-400 nm [14]. The spectrogram of the corona, with an increase in scale 1000 times, is highlighted in blue. The spectrum of the corona view corresponds to the electromagnetic radiation of atmospheric nitrogen when it is ionized. The mechanism of this process, in particular, can help to eliminate the defect or contamination of insulation. It follows from Fig. 1 that the spectrum of the corona has two pronounced maxima arising at 340 and $360 \mathrm{~nm}$. At the same time, in these ranges the strength of solar radiation is also great.

In order to be able to diagnose the insulator in daylight, in the modern electron-optical of UV-camera a range of $240-280 \mathrm{~nm}$ is used. In this range, solar radiation almost completely absorbs molecules of atmospheric ozone [3], and isolation can be observed with small hindrances or their absence.

Factors, influencing results of UV-inspection [13, 16]:

- $\quad$ wind speed;

- $\quad$ air humidity;

- ambient temperature;

- $\quad$ the distance to the surveyed object;

- $\quad$ viewing angle;

- $\quad$ Presence of precipitation;

- $\quad$ degree of insulation contamination [17]. 
This method of diagnostics has not been sufficiently studied yet and needs to be refined and verified in order to obtain more reliable results. For this purpose, experiments were carried out to solve the following problems:

- Check the camera's capabilities to measure the characteristics of the crown.

- Make sure that the results obtained are correct during the diagnostics in the power system.

\section{Analysis of experimental results}

We used guidelines [18] written specifically for laboratory work on a high-voltage test bench, which is located on the territory of the High Voltage Engineering Department.

\subsection{Calibration}

Using the camera, we received a combined image, processed from two matrices. An example of an image is shown in Fig. 1. The gray rectangle limits the area where the number of flashes is counted. For example, we can observe the crown at the end of the needle conductor and on its bends, as well as on the copper wire. At the bottom of the photo is information about the shooting mode, the current values of the gain and the number of flashes.

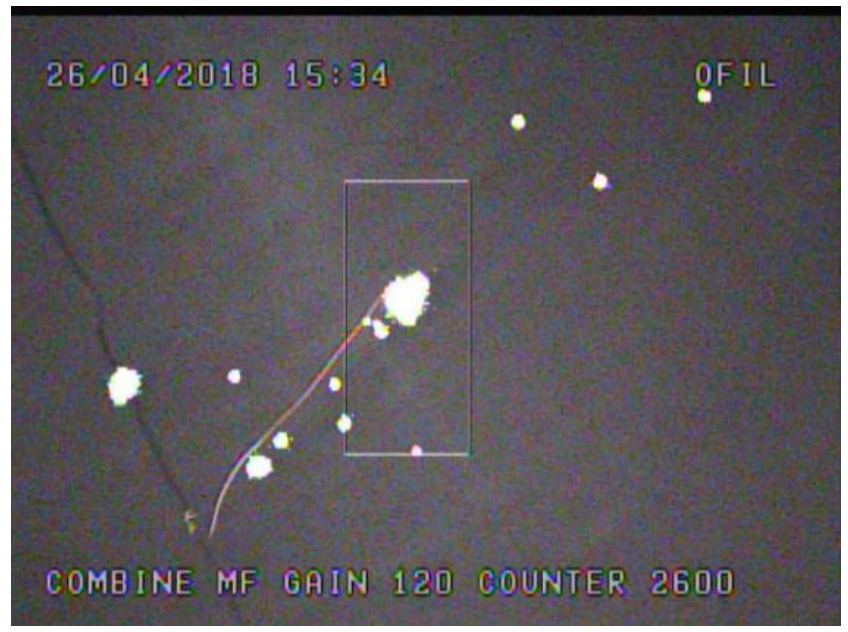

Fig. 1. Coronating needle conductor.

Before the experiments, the diagnostic device was calibrated. From the obtained data, it was found that with an increase in the sensitivity gain of the matrix, the error in the readings of the UV camera also increases.

\subsection{Determination of the minimum sensitivity by the UV camera}

For this experiment, an oscilloscope was used to measure the corona current and the power of the corona losses. At different magnification values: 40, 80, 120 and 200, the detection voltage of the corona of the UV camera and the oscilloscope differed for the needle conductor. Measurement of the corona discharge current allowed to fix the beginning of the corona discharge at a voltage of $35 \mathrm{kV}$, while the camera fixed the corona at a voltage of 32 $\mathrm{kV}$. But the advantage of the camera over the method based on measuring the corona current 
was reduced to a minimum when the observation object was changed to a wire where the detection voltage of the corona was the same: $35 \mathrm{kV}$.

\subsection{Determine the dependence of the UV camera readings on the survey distance}

The source of the crown was the needle guide. The dependence of the number of flashes on the shooting distance is clearly shown in Fig. 2.

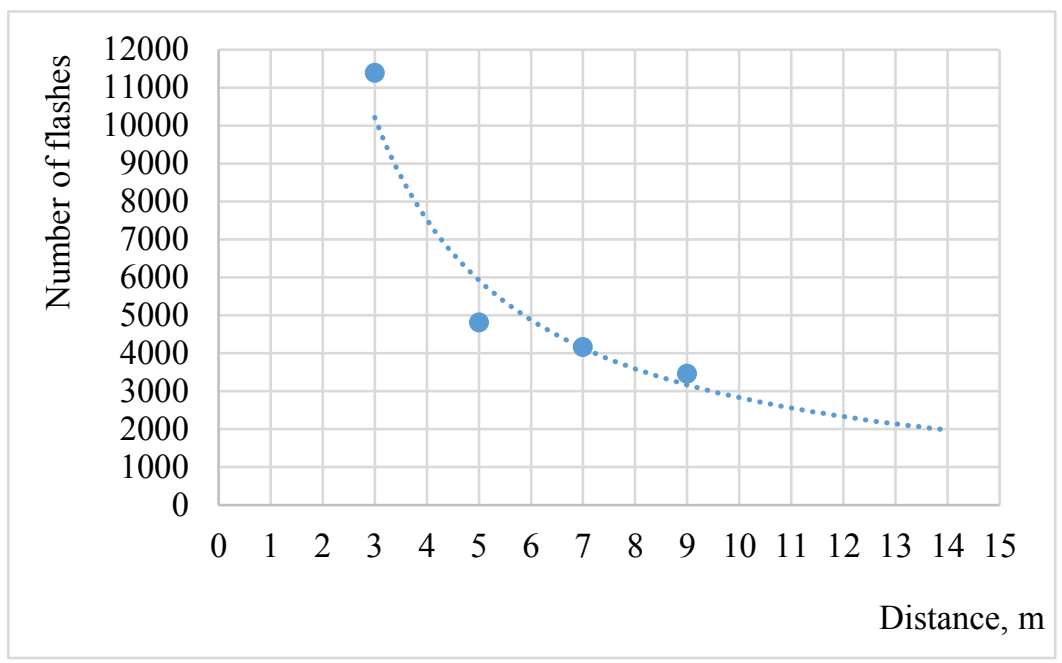

Fig. 2. Graph of the dependence of the number of flashes on the distance to the object.

The dependence of the number of flares on the distance to the object has a power-law character (Fig. 2). The difference between the UV camera readings for distances of 3 and 5 meters is the most significant: at a distance of 3 meters the number of recorded outbreaks was 2.4 times higher than the readings at a distance of 5 meters. At a subsequent distance from the object under examination, the readings decreased slightly. In practice, the UV inspection is usually carried out at a distance of not less than 5 meters from the facility, so the impact on the conclusion about UFD will be influenced by cases of shooting from a closer distance. The similar picture we can observe in [19-25].

\subsection{Comparison of the dependence of the UV chamber and the power loss on the corona on the voltage for a copper wire and a needle conductor.}

\subsubsection{Needle conductor}

The first experiment was carried out for a needle conductor with a gain of 120 . For different voltage values, 21 flashes were taken every 3 seconds (the refresh rate of the camera readings). 


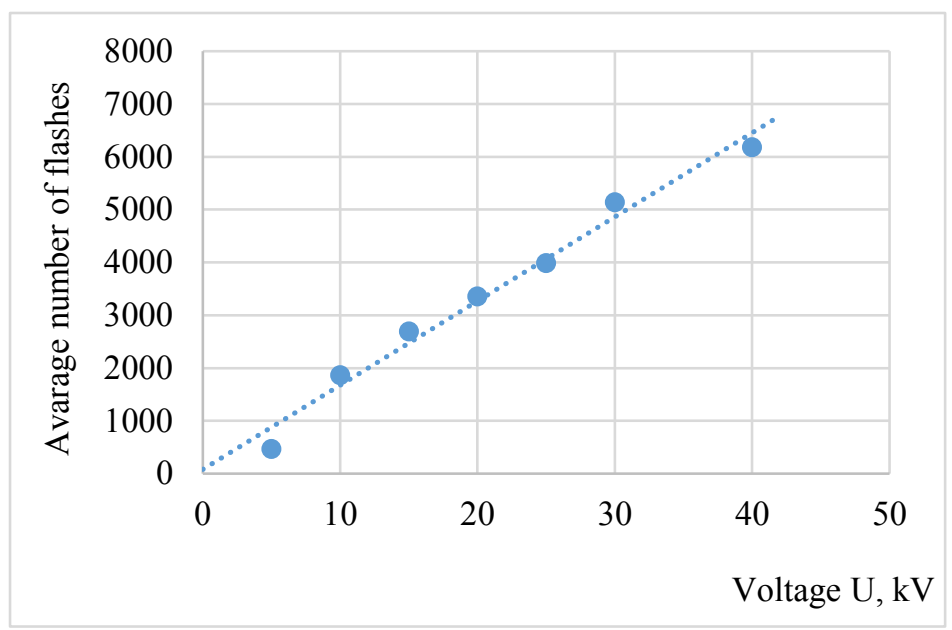

Fig. 3. Graph of the dependence cp. number of flashes from the voltage for the needle conductor at a gain of 120 .

The graph in Fig. 3 is linear. The voltage at which the corona discharges on the wire affected the characteristic of the corona from the needle conductor came at a voltage of 45 $\mathrm{kV}$, so the experiment was not conducted with a high voltage.

\subsubsection{Copper wire}

For this experiment, a copper wire with a cross section of $2 \mathrm{~mm}$ was used.

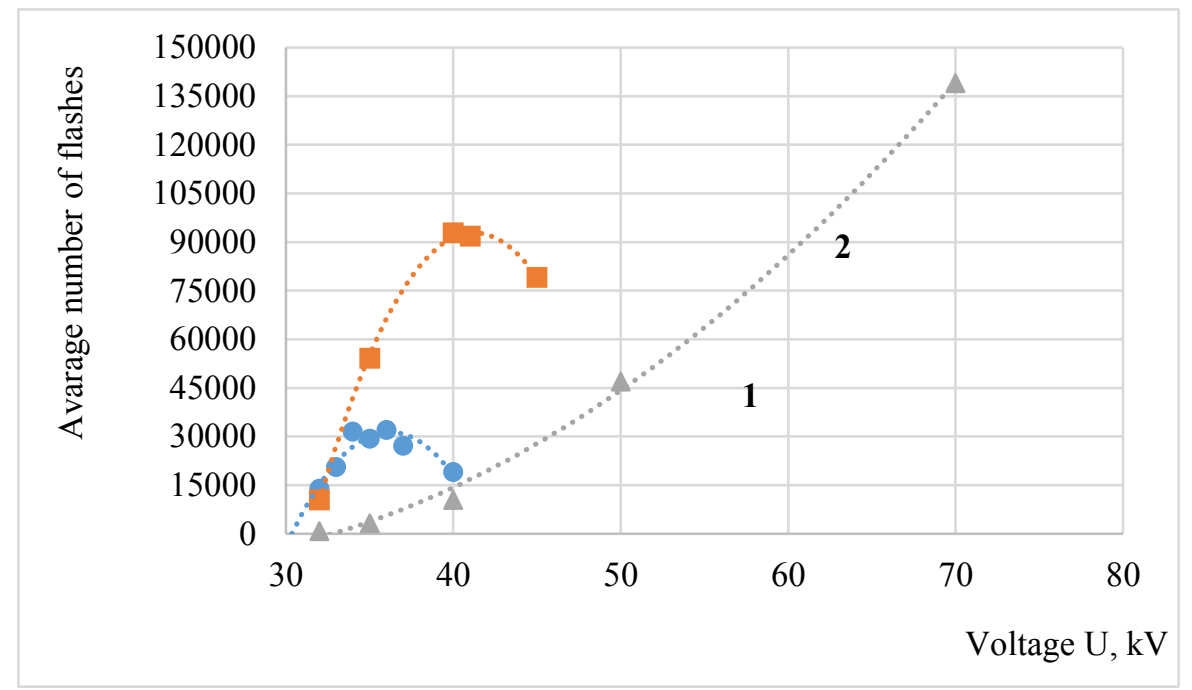

Fig. 4. Graphs of dependence of the number of flashes from the voltage for a copper wire at different gain factors: 1) 120,2) 80, 3) 40.

Analyzing the obtained graphs, we see that for the amplification factors 120 and 80, when a certain voltage is reached, an inflection appears, after which the number of flashes decreases. This phenomenon can be explained by the high sensitivity of the camera, which affects its selectivity: flashes on the matrix merge, and accordingly, the calculation of their 
number becomes incorrect. When the gain was reduced to 40 , the kink was not observed, but presumably it should occur at a high voltage, the achievement of which was not possible due to the limitations of the test bench.

It is also worth noting that with no gain the result will not be absolutely accurate, since at high values of sensitivity, flashes can merge, and at small values, it can not be detected.

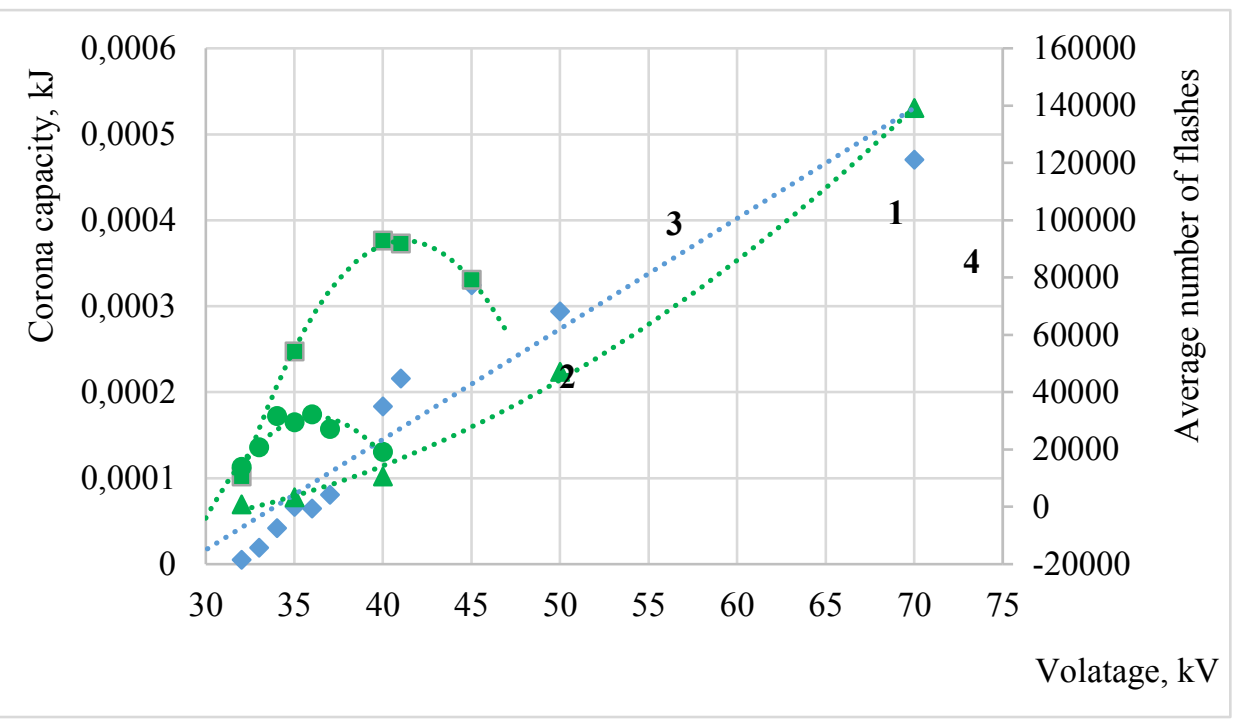

Fig. 5. Graphs of dependence cf. number of flares and power loss per crown (1) of the voltage for a copper wire at different gain factors: 2) 120,3) 80,4) 40 .

According to the graph in Fig. 5, the dependence of the power of losses on the corona on the voltage is linear, which corresponds to the theoretical curve.

\subsection{Determination of the dependence of the indications of the UV-camera on the voltage for a glass pendant insulator}

To approach the real conditions, an experiment was conducted with a glass suspension insulator. In the experiment, two plates of garland were used to reduce the stress of the appearance of the crown. Below is a photograph of the measurement of the number of flashes on an insulator with different gain factors. 


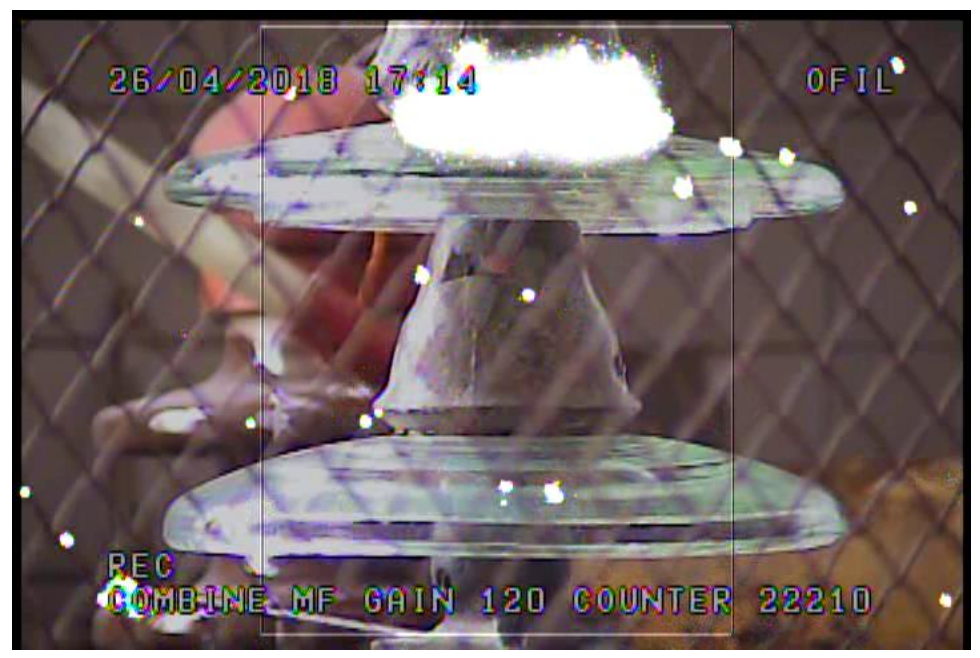

Fig. 6. Measurement of the number of flares for a glass insulator with a gain of 120 at a voltage of 70 $\mathrm{kV}$.

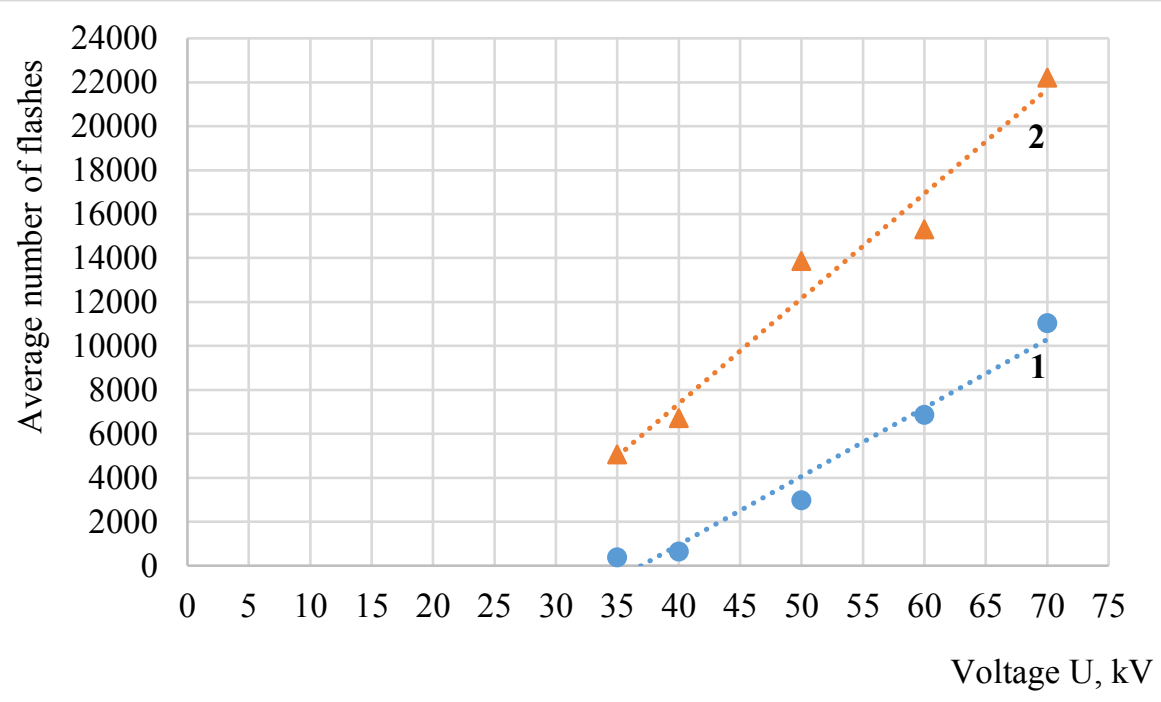

Fig. 7. Graph of the dependence cp. number of flashes from the voltage for different values of the gain factors: 1) 40; 2) 120 .

The graphs in Fig. 7 are linear in nature. A similar picture was observed for the needle conductor, which is the standard source of the corona, which confirms the correctness of the measurements.

\section{Conclusion}

According to the results of the experiments, it can be said that UV-inspection is a fairly good method for the rapid diagnosis of electrical equipment. But this method has its advantages and disadvantages, which must be taken into account when conducting the survey:

1. The UV camera has a high sensitivity, comparable with the sensitivity of the laboratory installation. 
2. The distance to the subject has a strong influence on the UV-camera, but with its increase, the number of flashes varies slightly. This fact allows you to conduct a survey from a certain distance without a strong scatter in the camera.

3. The camera has a limit of measuring the number of flashes per unit of time due to the resolution of the sensor, which requires a reduction in the gain, but at the same time, weaker signals are lost.

4. The curves of the dependence of the power of losses on the corona and the number of flashes on the voltage have a similar tendency, which makes it possible to bring the camera to the traditional physical quantity used, the power of the corona loss [20].

5. It is necessary to take into account the errors and peculiarities of the camera, which do not allow unambiguous interpretation of the result of the survey, as described in the instructions. Therefore, it is necessary to develop a methodology that takes into account these features.

\section{References}

1. R.S. Arbuzov, Modern methods of diagnostics of overhead power lines, Novosibirsk: Science, 136 (2009)

2. V.N. Lifanov, Electrical Insulation and Surge, Proc. allowance. Vl-c .: FSTU, 126 (2003)

3. Acquisition and interpretation of images of electrical discharges by the method of optical $U V$-flaw detection (PANATEST LLC).

4. Bin Ma. Effects of corona and ozone exposure on properties of polymeric materials for high voltage outdoor applications / Sweden, 125 (2011)

5. L.D. Ginzburg, Solid insulation of high-voltage structures for indoor installations. - SPb .: Energoatomizdat. St. Petersburg (1992)

6. A.G. Galkin, T.A. Nesenyuk, Control and Diagnostics of Power Line Insulators // Electrification of Transport, 6 (2013)

7. R.S. Arbuzov, A.G. Ovsyannikov, Journal PL-2018, 229-232(2018)

8. G.S. Fedorov, Method and measurement system for assessing the state of high-voltage insulators based on the analysis of partial discharges Kazan Russian State Library (2007)

9. Speech by V. Ostrovlyansky "The correct interpretation of the testimony of a UV flaw detector" at the "Dimrus-2018" conference.

10. P.M.Svi. Methods and tools for diagnosing high voltage equipment, - M .: Energoatomizdat, 240 (1992)

11. Scientific-technical and educational journal "Electromechanics" 1, 208 (2013)

12. S.M. Kutsenko, N.N. Klimov, V.I. Muratov, Bulletin of Tomsk Polytechnic University, 309 (2) (2006)

13. Production instruction of Lenenergo PJSC on the procedure for conducting ultraviolet examination of insulation of high-voltage lines and power lines. Revision 1 (2015)

14. Transmission line reference book $345 \mathrm{kV}$ and above / General electric company Published by Electric Power Research Institute, New York (1980)

15. F.D. Zheleznov, Yu.I. Plotnikov, V.A. Akulov, Railways of the World 4, 60-68 (2011)

16. A.G. Ovsyannikov, D.A. Brown, R.S. Arbuzov, V.M. Tolchin, News Electrical. 5(101), 60-62 (2016) 
17. Yu.N. Bocharov, S.M. Dudkin, V.V. Titkov, High voltage technique. High-voltage tests and measurements: textbook/. - SPb .: Polytechnic Publishing House. University, 210 (2013)

18. C. Lan, L. Lin, T. Mimi, B. Xingming, W. Liming, G. Zhicheng, Energy and power engineering 5 (2013)

19. S.A. Lopatkin, Guidelines for high-voltage tests. Tomsk Polytechnic University (2008)

20. M.A. Elistratova, N.M. Romanov, D.N. Goryachev, I.B. Zakharova, O.M. Sreseli, Semiconductors 51, 483-487 (2017), doi:10.1134/S1063782617040029

21. B.M. Broderick, V.Y. Chernyak, A.G. Smolin, O.S. Vasyutinskii, A.G. Suits, Chemical Physics Letters 635, 350-354 (2015), doi:10.1016/j.cplett.2015.07.021

22. E.M. Kantor, M.E. Gusakov, A.I. Chugunov, Monthly Notices of the Royal Astronomical Society 455, 739-753 (2016), doi:10.1093/mnras/stv2352

23. J. Vink, S. Broersen, A. Bykov, S. Gabici, Astronomy and Astrophysics 579, (2015), doi:10.1051/0004-6361/201424612

24. S.A. Balashev, P. Noterdaeme, V.V. Klimenko, P. Petitjean, R. Srianand, C. Ledoux, A.V. Ivanchik, D.A. Varshalovich, Astronomy and Astrophysics 575, (2015), doi:10.1051/0004-6361/201425553

25. A.A. Anfinogentova, M.N. Dudin, N.V. Lyasnikov, O.D. Protsenko, Economy of Region 14, 638-650 (2018), doi:10.17059/2018-2-24 